\title{
Characteristics of patients misdiagnosed with Alzheimer's disease and their medication use: an analysis of the NACC-UDS database
}

\author{
Joseph E Gaugler ${ }^{1 *}$, Haya Ascher-Svanum², David L Roth ${ }^{3}$, Tolulope Fafowora ${ }^{3}$, Andrew Siderowf ${ }^{4}$ \\ and Thomas G Beach ${ }^{5}$
}

\begin{abstract}
Background: This study compared individuals whose clinical diagnosis of Alzheimer's disease (AD) matched or did not match neuropathologic results at autopsy on clinical and functional outcomes (cognitive impairment, functional status and neuropsychiatric symptoms). The study also assessed the extent of potentially inappropriate medication use (using potentially unnecessary medications or potentially inappropriate prescribing) among misdiagnosed patients.

Methods: Longitudinal data from the National Alzheimer's Coordinating Center Uniform Data Set (NACC-UDS, 2005-2010) and corresponding NACC neuropathological data were utilized to compare 88 misdiagnosed and 438 accurately diagnosed patients.

Results: Following adjustment of sociodemographic characteristics, the misdiagnosed were found to have less severe cognitive and functional impairment. However, after statistical adjustment for sociodemographics, dementia severity level, time since onset of cognitive decline and probable AD diagnosis at baseline, the groups significantly differed on only one outcome: the misdiagnosed were less likely to be depressed/dysphoric. Among the misdiagnosed, $18.18 \%$ were treated with potentially inappropriate medication. An additional analysis noted this rate could be as high as $67.10 \%$.

Conclusions: Findings highlight the importance of making an accurate AD diagnosis to help reduce unnecessary treatment and increase appropriate therapy. Additional research is needed to demonstrate the link between potentially inappropriate treatment and adverse health outcomes in misdiagnosed AD patients.
\end{abstract}

Keywords: Alzheimer disease, Diagnosis, Misdiagnosis, Autopsy, Neuropathology

\section{Background}

Alzheimer's disease $(\mathrm{AD})$ is a progressive neurodegenerative disease and is the most common cause of dementia, accounting for about $60 \%$ of all cases [1]. The clinical diagnosis of $\mathrm{AD}$ is a challenging evaluation process that follows established clinical criteria and requires elimination of other potential causes for dementia [2,3]. Various studies have previously assessed the accuracy of the clinical diagnosis of AD based on autopsy results, or the "gold standard." A recent and comprehensive study showed that depending on

\footnotetext{
*Correspondence: gaug0015@umn.edu

${ }^{1}$ Center on Aging, School of Nursing, University of Minnesota, Minneapolis, MN, USA

Full list of author information is available at the end of the article
}

the permissiveness of clinical and neuropathologic criteria, sensitivity ranged from $70.9 \%$ to $87.3 \%$ and specificity ranged from $44.3 \%$ to $70.8 \%$ [4]. This and other studies found that between $12 \%$ and $23 \%$ of patients diagnosed with $\mathrm{AD}$ do not have sufficient $\mathrm{AD}$ pathology at autopsy to account for the presence of dementia ("misdiagnosed") [5-9].

The observed misdiagnosis rate may be partly driven by the fact that numerous conditions can mimic symptoms of $\mathrm{AD}$ [2]. Some of these conditions constitute other types of progressive dementias (e.g., frontotemporal dementia, vascular dementia and dementia with Lewy bodies) while others may be treatable and possibly reversible conditions (e.g., drug intoxication, depression, nutritional deficiencies,

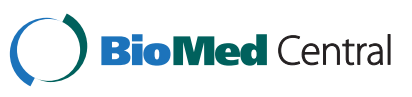


infectious diseases) [10,11]. In a recent retrospective analysis of clinical trials, $63 \%$ of deceased patients who were clinically diagnosed with $\mathrm{AD}$ while alive were found to have $\mathrm{AD}$ with other pathology [12]. In addition, older persons appear to have more etiologies than younger individuals which potentially attenuates the accuracy of clinical $\mathrm{AD}$ diagnosis [13]. Other studies have found higher levels of concordance between clinical and post-mortem diagnosis of $\mathrm{AD}$ but diminished diagnostic accuracy with other types of dementia [14,15].

Ruling out AD may result in changing patients' management plans that can lead to further evaluation and testing for the true underlying cause. Excluding AD may also enable appropriate treatment of the true underlying condition. A number of medications have been identified as potentially unnecessary for patients with frontotemporal dementia [16-20] and dementia with Lewy bodies $[21,22]$, whereas treatment with statins, antiplatelet agents and anticoagulants is deemed appropriate for patients with cerebrovascular disease.

At present limited information is available on the clinical, functional and socio-demographic characteristics of persons who have been misdiagnosed with $\mathrm{AD}$ based on neuropathologic results [23]. Similarly, it is unknown whether misdiagnosis of $\mathrm{AD}$ is associated with potentially unnecessary treatment or may result in patients not receiving treatments that are more appropriate for their conditions. To help address this knowledge gap we expanded on the study by Beach et al. [4] which identified individuals whose clinical diagnosis matched or mismatched their diagnosis per neuropathologic examination post-mortem. Using data collected as part of the National Alzheimer's Coordinating Center Uniform Data Set (UDS) (NACC-UDS) between 2005 and 2010, Beach and colleagues identified 88 participants misdiagnosed with $\mathrm{AD}$ and 438 participants accurately diagnosed with $\mathrm{AD}$ [5]. The goal of our study was to address two specific research questions: 1) When compared to accurately diagnosed $\mathrm{AD}$ patients, do misdiagnosed patients vary significantly on sociodemographic characteristics, health history, and key clinical and functional outcomes (cognitive impairment, functional status and neuropsychiatric symptoms); and 2) What is the extent of potentially inappropriate medication use among misdiagnosed patients?

\section{Methods}

\section{Subjects}

The National Alzheimer's Coordinating Center (NACC) [24] serves as the primary repository and data hub of the 34 past and present National Institute on Aging Alzheimer's Disease Centers (ADCs). Alzheimer's Disease Centers are located throughout the United States, are based in university medical centers, and are largely in urban areas [4]. Recruitment occurs through referrals from neurologists as well as community outreach efforts. The NACC Uniform Data Set (or NACC-UDS) is a publicly accessible, longitudinal database that includes standardized cognitive, behavioral, and functional data for each ADC participant based on their annual visits. The NACC-UDS was initiated in 2005. Of particular relevance to the current study, the NACC-UDS includes longitudinal data on persons with different etiologies of dementia as well as individuals not diagnosed with dementia. The procedure of AD clinical diagnosis (in living subjects) ranges from that of a consensus panel to a single physician according to each ADC's diagnostic protocol; however, each ADC generally adheres to standardized clinical criteria as outlined by the DSM-IV or NINDS-ADRDA guidelines [25]. The NACC-UDS provides systematic information on the following domains: demographics, behavioral status, cognitive testing, medical history, family history, clinical impressions, and diagnoses. For more detail on the construction of the NACC-UDS, please see Morris et al. [26]. Ethical approval for the current study was provided by the University of Minnesota Institutional Review Board (IRB\#1108E03546).

Participants in the current analysis were previously identified in Beach et al.'s study [4]. NACC-UDS data from 2005-2010 were considered for participants that had at least one UDS assessment, had died, and had brain autopsy results available $(n=1198)$. Of these individuals, 279 were excluded because they were considered "not demented" during regular UDS assessments or did not have data entered in key diagnostic entry fields (i.e., presence or absence of clinically probable $A D$ and CERAD plaque density or Braak stage) [4].

Of these remaining 919 individuals, two subgroups were the focus of the current analysis. Those in the accurately diagnosed group received a primary clinical diagnosis of probable AD based on NINDS-ADRDA criteria [26] and also received a neuropathological diagnosis/verification of $\mathrm{AD}$ per moderate or frequent density on the CERAD neuritic plaque density score [25] and a Braak neurofibrillary tangle stage of III-VI [27]. The classification of those accurately diagnosed with probable $\mathrm{AD}$ was based on a comprehensive analysis using various thresholds of CERAD neuritic plaque density score and Braak neurofibrillary tangle stages to find those with the greatest predictive value by Beach and colleagues $(n=438$; see p. 268). Those in the misdiagnosed group received a primary clinical diagnosis of probable $\mathrm{AD}$ but did not meet the aforementioned neuropathological threshold for $\mathrm{AD}$ at autopsy (i.e., a moderate or frequent CERAD density score or a Braak neurofibrillary tangle stage of III-VI; $n=88$ ). The primary neuropathologic diagnoses of those in the misdiagnosed group included primary neuropathologic diagnosis of $\mathrm{AD}$ despite a low level of AD histopathology $(n=17)$, tangle-only dementia or 
agryophilic grain disease $(\mathrm{n}=15)$, frontotemporal lobar degeneration $(n=15)$, cerebrovascular disease $(n=10)$, Lewy body disease, with or without $\mathrm{AD}(\mathrm{n}=9)$, hippocampal sclerosis, with or without AD $(n=9)$, progressive supranuclear palsy $(n=3)$, corticobasal degeneration $(n=2)$, neuroaxonal dystrophy/Hallevorden-Spatz-like condition $(n=2)$, and miscellaneous $(n=6 ; 1$ case each of amyloid angiopathy, "small vessel disease," "TDP-43 proteinopathy," limbic encephalitis, Rosenthal fiber encephalopathy, "clinical dementia, no neuropathological substrate") [4].

In the original Beach et al. analysis, subjects were diagnosed with possible AD $(n=126)$ using NINDS-ADRDA guidelines [26]. These individuals had a neuritic plaque density average of $2(\mathrm{SD}=1.2 ; 0=$ none; $1=$ sparse; $2=$ moderate; and $3=$ frequent) and a Braak stage mean of $4.2(\mathrm{SD}=1.6)$ at death. Another subgroup of participants $(n=271)$ was classified as not having either probable or possible AD based on NINDS-ADRDA guidelines, and of these a substantial proportion had a neuropathological diagnosis of $\mathrm{AD}(\mathrm{n}=107$; "false negatives") or other neuropathological diagnoses: frontotemporal lobar degeneration $(n=60)$, Lewy body disease with or without AD $(n=31)$, Creutzfeldt-Jakob disease and other prior encephalopathies $(n=23)$, progressive supranuclear palsy $(\mathrm{n}=18)$, tangle-only dementia or argyrophilic grain disease $(n=9)$, corticobasal degeneration $(n=8)$, Pick's disease $(n=6)$, cerebrovascular disease $(n=6)$, hippocampal sclerosis with or without AD $(n=2)$, amyotrophic lateral sclerosis $(n=2)$, and miscellaneous (1 case each of neuronal intermediate filament disease, "leukodystrophy," and cerebellar atrophy; $n=3$ ). As one of the aims of the current study was to examine whether potentially inappropriate medication use occurred among those who were misdiagnosed as having probable $\mathrm{AD}[4]$, those in the possible AD group as well as the false and true negatives in the original Beach et al. analysis were excluded.

\section{Measures}

The following measures available at the first time a probable AD diagnosis was recorded in the NACC-UDS were included. Socio-demographic/background characteristics included age, gender, race (Caucasian vs. nonCaucasian), education (years), marital status (married/ living as married or not), and living alone (yes/no). Health history and conditions included any family history of dementia (a parent with dementia, a sibling with dementia and number of other relatives with dementia), health history (any history of cardiovascular disease, cerebrovascular disease, parkinsonian features, other neurologic conditions, medical/metabolic conditions), etiology of $\mathrm{AD}$ ( $\mathrm{AD}$ only or a mixed etiology), and living in a nursing home (yes/no). Since individuals who participated in the NACC-UDS may have received an
Alzheimer's disease diagnosis prior to enrollment (and time of diagnosis prior to NACC-UDS was not recorded), two additional variables were included to address potential heterogeneity of disease stage. A dichotomous variable identified those who were recorded as having or not having a probable AD diagnosis at baseline in the NACC-UDS. Informants also reported on participants' years since onset of cognitive decline at the initial NACC-UDS visit. Severity of cognitive impairment was measured with the Mini-Mental Status Examination/MMSE (mean score and level of cognitive impairment: normal $>24$, mild 21-24, moderate 10-20 and severe $<=9$ ) [28]. Functional status was assessed with the Functional Assessment Questionnaire/FAQ (total score and proportion of patients with impaired functioning, per total score of 9 or above) [29]. Neuropsychiatric symptomatology was measured with the Neuropsychiatric Inventory Questionnaire/NPI-Q (total score) [30]. Due to extensive missing data on the Geriatric Depression Scale/GDS [31] in the accurately diagnosed group (approximately 30\%), depression was assessed using other available measures: a) proportion of depressed subjects who had a "yes" response per the NPI-Q depression or dysphoria item completed by informants of the NACC-UDS participant; and b) severity of depression among subjects identified as depressed/dysphoric on the NPI-Q, also completed by informants.

\section{Use of potentially inappropriate medications}

Data on medication use at or following the first assessment which subjects' probable AD diagnosis was recorded in the NACC-UDS were considered. The identification of potentially inappropriate medications (the use of potentially unnecessary medications or potentially inappropriate prescribing) was deemed feasible for 3 specific subgroups within the misdiagnosed group based on prior research: those diagnosed post-mortem with either frontotemporal dementia (FTD, $\mathrm{n}=18$ ), dementia with Lewy bodies (DLB, $n=9)$, or cerebrovascular disease $(n=10)[4,18,20,21,32]$. Prior to start of the analysis, a neurologist at Eli Lilly and Company created an appropriate and potentially inappropriate medication matrix list based on the NACC-UDS medication checklist. Classification of potentially inappropriate medications was then based on clinical treatment guidelines and available research evidence.

The use of acetylcholinesterase inhibitors was considered potentially inappropriate for subjects whose true diagnosis was FTD at autopsy [20]. A previous study evaluating donepezil in the treatment of FTD relative to matched, untreated FTD patients over six months found that a third of the treated patients experienced increased disinhibited or compulsive acts, which abated with discontinuation of the medication [33]. These and similar observations have prompted a general recommendation to avoid 
acetylcholinesterase inhibitors in FTD $[16,17,33]$. Recent randomized controlled trials also suggest that memantine lacks efficacy in the treatment of FTD [34].

The use of antipsychotics, except for quetiapine or clozapine, is considered potentially inappropriate for patients with dementia with Lewy bodies (DLB) as these individuals may experience severe side effects or fatal complications if behavioral symptoms are treated with antipsychotic drugs [35]. Patients with DLB are particularly sensitive to developing extrapyramidal symptoms and potentially fatal complications of neuroleptic sensitivity, which affects approximately 50\% of DLB patients. Administering antipsychotic medications for behavioral symptoms to patients with DLB can potentially result in serious neuroleptic sensitivity reactions which are associated with significantly increased morbidity and mortality $[21,22]$.

To further assess whether participants in the misdiagnosed group were subject to potentially inappropriate prescribing, we determined the number of participants in the misdiagnosed group with cerebrovascular disease who did not use statins, antiplatelet agents or anticoagulants. These medications are considered appropriate for those with cerebrovascular disease [33]. Thus, the use of potentially inappropriate medication was defined as (a) the use of an anti-dementia drug by those whose true diagnosis was found at autopsy to be FTD, or (b) the use of an antipsychotic drug by those whose true diagnosis was found at autopsy to be DLB, or (c) not being treated with statins, antiplatelet agents or anticoagulants by those whose true diagnosis was found at autopsy to be cerebrovascular disease.

As a sensitivity analysis, the use of potentially inappropriate medications was also assessed using a broader definition which included the above criteria or the use of an anti-dementia drug by any of the misdiagnosed patients (i.e., not confined to those found to have FTD at autopsy). The rationale for broadening the definition was that anti-dementia drugs have an approved indication for the treatment of $A D$, whereas there is a lack of evidence-based support for non-AD/misdiagnosed individuals receiving such pharmacological intervention [36]. The "off label" use of anti-dementia drugs was, therefore, considered potentially unnecessary for the misdiagnosed subjects in the study. Notably, there is an anti-dementia drug (rivastigmine) that is indicated for dementia due to Parkinson's disease but none of the misdiagnosed in the current study were diagnosed with this condition.

\section{Analysis}

The principal objective of this analysis was to statistically compare the misdiagnosed and accurately diagnosed groups on sociodemographics, health history, and clinical and functional outcomes (cognitive impairment, functional status, and neuropsychiatric symptoms) as assessed at the first UDS assessment for which a clinically probable AD diagnosis was recorded in the NACC-UDS. Group comparisons for all variables were first conducted using unadjusted bivariate analysis (e.g., unadjusted logistic or multinomial regressions for categorical variables, T-tests for continuous measures). To assess whether the groups significantly differed on key outcomes (severity of cognitive impairment, functional status, and neuropsychiatric symptoms) when their core demographic characteristics, time since onset of cognitive decline and dementia severity were held constant, we conducted two sets of adjusted analyses: one controlling for participants' key sociodemographic characteristics (age, education, gender, race, and marital status) and the second controlling for the aforementioned sociodemographics as well as time since onset of cognitive decline, a probable $\mathrm{AD}$ diagnosis at baseline of the NACC-UDS (yes/no), and dementia severity (categorical, as assessed by MMSE levels noted above). The second adjusted analysis was performed because dementia severity, the presence of probable $\mathrm{AD}$ diagnosis at baseline, and time since symptom onset are core clinical characteristics of AD and are correlated with other key clinical and functional outcomes. Analyses of covariance were used for continuous outcomes and logistic or multinomial logistic regression analyses were used for categorical outcomes.

An additional study objective was to examine potentially inappropriate medication use by the misdiagnosed group. Using data on medication use at or following the first assessment when subjects' probable $\mathrm{AD}$ diagnosis was recorded in NACC-UDS, we identified potentially inappropriate medication use for all misdiagnosed participants. SAS version 9.3 [37] was used to extract data and perform all analyses.

\section{Results}

\section{Sociodemographic background characteristics and health} history

Participant socio-demographic characteristics, health history, and bivariate comparisons between the misdiagnosed and accurately diagnosed groups are presented in Table 1. Participants in the misdiagnosed group were significantly $(\mathrm{p}<.05)$ older than those in the accurately diagnosed group (83.52 years vs. 78.72 years, respectively), were more likely to live alone $(17.05 \%$ vs. $4.11 \%$, respectively), and were less likely to be married (56.82\% vs. $71.00 \%$, respectively) at the time of study entry or first AD diagnosis. For all other socio-demographic characteristics, the two diagnostic groups did not significantly differ. A significantly higher proportion of individuals in the misdiagnosed group had a history of a cardiovascular condition (47.13\%) than did those in the accurately diagnosed group (31.49\%). Those in the 
Table 1 Socio-demographic and clinical characteristics of the misdiagnosed and accurately diagnosed groups: unadjusted comparisons

\begin{tabular}{|c|c|c|c|c|c|}
\hline & $\begin{array}{l}\text { Misdiagnosed } \\
\mathrm{N}=88\end{array}$ & $\begin{array}{c}\text { Accurately } \\
\text { diagnosed } \mathrm{N}=438\end{array}$ & $\begin{array}{l}\text { Parameter } \\
\text { estimate }\end{array}$ & $\begin{array}{l}\text { Unadjusted OR } \\
(95 \% \mathrm{Cl})\end{array}$ & $p$ value \\
\hline \multicolumn{6}{|l|}{ Sociodemographic characteristics } \\
\hline Age, Mean \pm SD & $83.52 \pm 10.31$ & $78.72 \pm 10.27$ & -4.80 & & $<.0001$ \\
\hline Years of education, Mean \pm SD & $14.53 \pm 3.56$ & $14.90 \pm 3.34$ & 0.37 & & .3528 \\
\hline Gender, N,\% male & $52,59.09$ & $254,57.99$ & & $0.96(0.60,1.52)$ & .8490 \\
\hline Race, $N, \%$ minority & $5,5.68$ & $24,5.48$ & & $0.96(0.36,2.59)$ & .9388 \\
\hline \multicolumn{6}{|l|}{ Race } \\
\hline White, N/\% & $83,94.32$ & $414,94.52$ & & (Ref) & \\
\hline Black, N/\% & $3,3.41$ & $17,3.88$ & & $1.14(0.33,3.97)$ & .8414 \\
\hline Asian, N/\% & $1,1.14$ & $4,0.91$ & & $0.80(0.09,7.27)$ & .8444 \\
\hline Other, N/\% & $1,1.14$ & $3,0.68$ & & $0.60(0.06,5.85)$ & .6614 \\
\hline \multicolumn{6}{|l|}{ Unknown, N,\% } \\
\hline Marital status, married/living as married, N,\% & $50,56.82$ & $311,71.00$ & & $1.86(1.16,2.98)$ & .0095 \\
\hline Living alone, $\mathrm{N}, \%$ & $15,17.05$ & $18,4.11$ & & $0.21(0.10,0.43)$ & $<.0001$ \\
\hline \multicolumn{6}{|l|}{ Health history and conditions } \\
\hline \multicolumn{6}{|l|}{ Family history of dementia } \\
\hline Number of "Other demented relatives," Mean \pm SD & $0.41 \pm 0.80$ & $0.63 \pm 1.20$ & 0.22 & & .1971 \\
\hline Father or mother with dementia, N,\% & $23,32.86$ & $183,47.78$ & & $1.87(1.09,3.20)$ & .0225 \\
\hline Any sibling with dementia, N,\% & $4,4.55$ & $8,1.83$ & & $0.39(0.12,1.33)$ & .1339 \\
\hline \multicolumn{6}{|l|}{ Health history } \\
\hline Cardiovascular condition, N,\% & $41,47.13$ & $137,31.49$ & & $0.52(0.32,0.82)$ & .0055 \\
\hline Cerebrovascular condition N,\% & $18,20.69$ & $66,15.28$ & & $0.69(0.39,1.24)$ & .2127 \\
\hline Parkinsonian features, N,\% & $9,10.23$ & $39,8.97$ & & $0.86(0.40,1.86)$ & .7087 \\
\hline Other neurologic conditions, N,\% & $17,20.00$ & $87,20.67$ & & $1.04(0.58,1.86)$ & .8908 \\
\hline Medical/metabolic conditions, N, $\%$ & $80,90.91$ & $361,82.61$ & & $0.48(0.22,1.02)$ & .0574 \\
\hline AD only vs. mixed etiology & $56,63.64$ & $298,68.04$ & & $0.82(0.51,1.33)$ & .4225 \\
\hline Living in nursing home, $N, \%$ & $13,14.77$ & $85,19.41$ & & $1.39(0.74,2.62)$ & .3103 \\
\hline Time since onset of cognitive decline (years) & $6.30 \pm 3.76$ & $7.81 \pm 4.01$ & 1.51 & & .0016 \\
\hline Probable AD diagnosis at baseline & $65,73.86$ & $412,94.06$ & & $5.61(3.02,10.41)$ & $<.0001$ \\
\hline \multicolumn{6}{|l|}{ Severity of cognitive impairment } \\
\hline MMSE, Mean \pm SD & $19.46 \pm 7.66$ & $12.93 \pm 9.03$ & -6.53 & & $<.0001$ \\
\hline Normal (>24): N,\% & $22,25.00$ & $33,7.53$ & & 1.00 (Ref) & \\
\hline Mild (21-24): N,\% & $23,26.14$ & $67,15.30$ & & $1.94(0.95,3.98)$ & .0699 \\
\hline Moderate (10-20): N,\% & $24,27.27$ & $136,31.05$ & & $3.78(1.89,7.55)$ & .0002 \\
\hline Severe $(<=9)$ : $N, \%$ & $11,12.5$ & $137,31.28$ & & $8.30(3.67,18.81)$ & $<.0001$ \\
\hline Missing: N,\% & $8,9.1$ & $65,14.84$ & & $5.42(2.18,13.48)$ & .0003 \\
\hline \multicolumn{6}{|l|}{ Functional status } \\
\hline Functional activities questionnaire (FAQ), total score, Mean $\pm S D$, & $20.96 \pm 8.52$ & $24.52 \pm 7.10$ & 3.55 & & $<.0001$ \\
\hline Impaired level of functioning, $\mathrm{FAQ}>=9, \mathrm{~N}, \%$ & $75,88.24$ & $405,95.74$ & & $3.0(1.33,6.75)$ & .0080 \\
\hline
\end{tabular}




\section{Table 1 Socio-demographic and clinical characteristics of the misdiagnosed and accurately diagnosed groups: unadjusted comparisons (Continued)}

\begin{tabular}{|c|c|c|c|c|c|}
\hline \multicolumn{6}{|l|}{ Neuropsychiatric symptoms } \\
\hline $\begin{array}{l}\text { Neuropsychiatric inventory questionnaire (NPI-Q), total } \\
\text { score, Mean } \pm \text { SD }\end{array}$ & $4.84 \pm 4.46$ & $6.18 \pm 5.35$ & 1.35 & & .0364 \\
\hline Patients with depression and dysphoria (NPI-Q), N,\% & $16,20.25$ & $145,37.08$ & & $2.32(1.29,4.17)$ & .0048 \\
\hline $\begin{array}{l}\text { Severity level for patients with depression and dysphoria } \\
\text { (NPI-Q), Mean } \pm \text { SD }\end{array}$ & $1.56 \pm 0.73$ & $1.40 \pm 0.62$ & -0.16 & & .3276 \\
\hline
\end{tabular}

NOTE: $\mathrm{Cl}=$ Confidence interval, $\mathrm{SD}$ = standard deviation, $\mathrm{AD}$ = Alzheimer's disease; MMSE = Mini Mental Status Examination.

accurately diagnosed group had experienced a significantly longer time since onset of cognitive decline than those in the misdiagnosed group (7.81 vs. 6.30 years, respectively) and were more likely to have a probable $\mathrm{AD}$ diagnosis at baseline $(94.06 \%$ vs. $73.86 \%$, respectively). For all other health history and condition variables the two groups did not significantly differ.

\section{Severity of cognitive impairment and outcomes}

Table 1 also provides detail on severity of cognitive impairment, functional status, and neuropsychiatric symptoms (including depression). Individuals in the misdiagnosed group scored significantly $(\mathrm{p}<.05)$ higher/ better on the MMSE (19.46) than those in the accurately diagnosed group (12.93), with a lower percentage of misdiagnosed participants scoring within the "severe" category ( $12.5 \%$ vs. $31.28 \%$, respectively). The misdiagnosed group also appeared less functionally impaired on the FAQ, on average, and a smaller proportion scored above the impaired clinical threshold than those in the accurately diagnosed group ( $88.24 \%$ vs. $95.74 \%$, respectively). The misdiagnosed also had lower/better average neuropsychiatric scores on the NPI-Q than those in the accurately diagnosed group (4.84 vs. 6.18, respectively). A higher percentage of those in the accurately diagnosed group had depression/dysphoria as measured on the NPI-Q item than those in the misdiagnosed group ( $37.08 \%$ vs. $20.25 \%$, respectively), but the two groups did not differ on the NPI-Q severity of depression indicator.

\section{Adjusted analyses}

We conducted a series of analyses to determine whether the observed group differences in clinical and functional variables were maintained following: a) adjustments for key socio-demographics (age, gender, race, marital status and education); and b) adjustments for key socio-demographics, dementia severity level (MMSE categorical scores), time since onset of cognitive decline, and whether probable $\mathrm{AD}$ diagnosis was recorded at baseline (see Table 2). Following adjustments for sociodemographic characteristics only, the results paralleled those of the unadjusted bivariate comparisons; individuals in the misdiagnosed group had significantly $(\mathrm{p}<.05)$ higher/better MMSE scores, had significantly lower/better FAQ scores, and were less likely to have depression/dysphoria on the NPI-Q. The NPI-Q total score no longer varied significantly following the adjustment of sociodemographic characteristics.

When including MMSE, time since onset of cognitive decline and probable $\mathrm{AD}$ diagnosis at baseline along with sociodemographics as covariates (see Table 2) the two groups were found to statistically differ on only one outcome: depression. A lower proportion of participants in the misdiagnosed group was found to have depression/dysphoria on the NPI-Q ( $\mathrm{p}=.0183)$.

We repeated the adjusted models with one sociodemographic variable as an outcome: living alone, as it is apt to have clinical ramifications in our cognitively impaired sample. The misdiagnosed group was more likely to live alone than those in the accurately diagnosed group $(\mathrm{OR}=.26$, $95 \% \mathrm{CI}=.11, .60, p=.0018)$ after adjusting for sociodemographic variables only. However, there was no significant difference in living alone between the misdiagnosed and accurately diagnosed groups after adjusting for sociodemographic variables, MMSE, time since onset of cognitive decline and probable $\mathrm{AD}$ diagnosis at baseline $(\mathrm{OR}=.47$, $95 \% \mathrm{CI}=.18,1.24, \mathrm{p}=.1269$ ).

\section{Medication use in the misdiagnosed group}

Results on medication use among the 88 misdiagnosed subjects were based on 145 observations at or after a probable AD diagnosis was recorded in the NACC-UDS. Among the misdiagnosed subjects, $18.18 \%$ (16 of 88) were on a potentially inappropriate medication regimen. When using the broader definition of potentially inappropriate medication, this rate increased to $67.1 \%$ (59 of 88). Among the misdiagnosed subjects in the FTD, DLB, or cerebrovascular subgroups, $43.2 \%$ (16 of 37) were classified as being on a potentially inappropriate medication regimen. This is based on pooling the following results: a) $55.5 \%$ of misdiagnosed subjects who were identified at autopsy as having FTD (10 of 18) were treated with acetylcholinesterase inhibitors or glutamate blockers; b) $11.1 \%$ of misdiagnosed subjects who were identified at autopsy as having DLB ( 1 of 9 ) were treated 
Table 2 Functional and clinical outcomes of the misdiagnosed and accurately diagnosed groups: adjusted comparisons

\begin{tabular}{|c|c|c|c|c|c|c|}
\hline & $\begin{array}{l}\text { Parameter } \\
\text { estimate }\end{array}$ & $\begin{array}{l}\text { Adjusted } \mathrm{OR}^{\mathrm{a}} \\
(95 \% \mathrm{Cl})\end{array}$ & $p$ value & $\begin{array}{l}\text { Parameter } \\
\text { estimate }\end{array}$ & $\begin{array}{l}\text { Adjusted OR } \\
\quad(95 \% \mathrm{Cl})\end{array}$ & $\mathrm{p}$ value \\
\hline \multicolumn{7}{|l|}{ Severity of cognitive impairment } \\
\hline MMSE (Mean) & -5.90 & & $<.0001$ & & & \\
\hline Normal (>24) & & 1.00 (Ref) & & & & \\
\hline Mild (21-24) & & $2.20(1.04,4.65)$ & .0388 & & & \\
\hline Moderate (10-20) & & $4.27(2.07,8.80)$ & $<.0001$ & & & \\
\hline Severe $(<=9)$ & & $8.16(3.48,19.15)$ & $<.0001$ & & & \\
\hline Missing & & $5.93(2.30,15.30)$ & .0002 & & & \\
\hline \multicolumn{7}{|l|}{ Functional status } \\
\hline $\begin{array}{l}\text { Functional activities questionnaire } \\
\text { (FAQ), total score (Mean) }\end{array}$ & 3.24 & & .0003 & -0.90 & & .1823 \\
\hline Impaired level of functioning, $\mathrm{FAQ}>=9$ & & $2.56(1.11,5.93)$ & .0281 & & $1.09(0.40,2.92)$ & .8703 \\
\hline \multicolumn{7}{|l|}{ Neuropsychiatric symptoms } \\
\hline $\begin{array}{l}\text { Neuropsychiatric inventory questionnaire } \\
\text { (NPI-Q), total score (Mean) }\end{array}$ & 0.81 & & .2018 & 0.55 & & .4222 \\
\hline $\begin{array}{l}\text { Patients with depression and dysphoria } \\
\text { (NPI-Q) }\end{array}$ & & $1.99(1.09,3.63)$ & .0251 & & $2.15(1.14,4.07)$ & .0183 \\
\hline $\begin{array}{l}\text { Severity level for patients with depression } \\
\text { and dysphoria (NPI-Q) }\end{array}$ & -0.18 & & .2697 & -0.21 & & .2327 \\
\hline
\end{tabular}

NOTE: $\mathrm{Cl}=$ Confidence interval, $\mathrm{SD}$ = standard deviation, MMSE = Mini Mental Status Examination; Analyses of covariance were used for continuous outcomes. ${ }^{a}$ Adjusted for age (continuous - in years), education (continuous - in years), gender, race (white vs. minority), and marital status (married vs. other).

${ }^{\mathrm{b}}$ Adjusted for age (continuous - in years), education (continuous - in years), gender, race (white vs. minority), marital status (married vs. other), dementia severity (MMSE), time since onset of cognitive decline, and probable AD diagnosis at Time 1.

with an antipsychotic medication (olanzapine); and c) $50 \%$ of misdiagnosed subjects who were identified at autopsy as having a cerebrovascular disease (5 of 10) were not treated with a statin, antiplatelet agent, or anticoagulant (the treatments considered appropriate for such conditions). The overall percentage of those in the misdiagnosed group who were treated with an antidementia drug at the time a probable $\mathrm{AD}$ was recorded in the NACC-UDS or thereafter was $64.8 \%$.

\section{Discussion}

This study compares characteristics of persons with an inaccurate $\mathrm{AD}$ diagnosis (i.e., a clinical diagnosis of $\mathrm{AD}$ but no neuropathological verification of AD) and an accurate AD diagnosis (those with a matching clinical and neuropathological diagnosis of AD at autopsy). Similar to other recent studies, the current analysis found that the misdiagnosed and accurately diagnosed groups significantly differed on several clinical and functional outcomes even after controlling for sociodemographic characteristics. When compared to the accurately diagnosed group, the misdiagnosed group was significantly older, less likely to be married, more likely to live alone, and more likely to have a history of cardiovascular conditions [23]. The misdiagnosed group also had a less severe illness profile in terms of dementia severity, family history of dementia, functional status and neuropsychiatric symptoms (including the presence of depression or dysphoria). This may have been due to the groups' variation in their dementia trajectories; individuals in the accurately diagnosed group had experienced cognitive decline for approximately $1 \frac{1}{2}$ years longer, on average, than those in the misdiagnosed group. Similarly, a higher proportion of individuals in the accurately diagnosed group had a probable AD diagnosis at baseline than those in the misdiagnosed group. The groups did not, however, significantly differ on neuropsychological symptoms (including severity of depression) following adjustment of core sociodemographic characteristics. Interestingly, although the misdiagnosed patients were older, they had a shorter duration of symptoms and thus the onset of their decline was at 77 years of age vs. 71 years of age for accurately diagnosed patients. The younger age of onset may predispose these patients to less complicated pathology whereas the misdiagnosed, because of their older age, may be more vulnerable to other conditions (e.g., cardiovascular) [23] that can masquerade as AD. Similarly, one reason for misdiagnosis occurring among patients with cardiovascular conditions is due to multiple pathologies occurring at autopsy, which is a common occurrence even in fairly restricted clinical trial samples of AD patients [12]. 
Importantly, when group comparisons were adjusted for age, gender, race, marital status, education, dementia severity, time since onset of cognitive decline and whether one had a probable AD diagnosis at baseline the two groups no longer differed significantly on any clinical or functional measure except for a single depression parameter. Those misdiagnosed were less likely to have depression or dysphoria when compared to the accurately diagnosed group. This depression-related finding should be evaluated with caution, as it was based on a single item and the groups did not significantly differ on the severity of depression parameter. Considering the non-specific nature of clinical diagnosis of dementia symptoms [4], current evaluation processes of AD diagnosis may not enable clinicians to differentiate misdiagnosed and accurately diagnosed patients in routine practice when patients have similar levels of cognitive impairment, socio-demographics and time since symptom onset. This scenario would, however, be different once these patients are not of similar dementia severity level or time since symptom onset as suggested in our adjusted models of sociodemographic characteristics only (as those in the misdiagnosed group tended to have a more recent time since symptom onset as well as less severe cognitive, functional and neuropsychiatric impairment).

The findings also suggested that $18.2 \%$ of individuals in the misdiagnosed group were on a potentially inappropriate medication regimen. This percentage may be clinically meaningful as such practice could adversely and inordinately influence misdiagnosed patients' health outcomes and lead to increased burden to patients, their caregivers, their physicians and healthcare payers. Moreover, when using a broader definition of "potentially inappropriate medication" the rate could be as high as $67.1 \%$. The latter finding appears driven by the $64.8 \%$ of misdiagnosed patients who were treated with anti-dementia drugs. The potential personal and economic ramifications of such extensive "off label" use of anti-dementia drugs are unclear and will require future study. It is important to note that although anti-dementia drugs are indicated for the treatment of $\mathrm{AD}$, treating those in the misdiagnosed group with an acetylcholinesterase inhibitor or a glutamate blocker is likely not inappropriate in all circumstances as such medications are often the practical and pragmatic therapeutic strategy for individual clinicians. Patients with non-AD dementias may also have a positive response to acetylcholinesterase inhibitors or glutamate blockers in some instances. Nonetheless, an accurate clinical/in vivo diagnosis is necessary in order to tailor treatment of actual underlying conditions rather than broad non-specific clinical syndromes.

The current study helps to highlight the importance of making an accurate diagnosis of AD in clinical practice. Improving diagnostic accuracy in clinical settings, and especially ruling out $\mathrm{AD}$, may help reduce unnecessary treatment as well as increase the administration of appropriate therapy for patients' conditions. There are growing efforts to improve diagnostic accuracy of $\mathrm{AD}$, including the use of biomarker testing and especially biomarkers with evidence for compelling negative predictive value (i.e., when a patient's test is negative it is most likely correct) $[2,38,39]$. Given the clinical complexity of distinguishing between potentially misdiagnosed and accurately diagnosed patients as demonstrated by our empirical results, biomarkers may provide a useful tool to clinicians to avoid or minimize possible misdiagnosis. Recent studies have found, for example, that the knowledge of beta amyloid positron emission tomography scan results can lead to substantial changes to clinicians' diagnoses and intended management plans $[40,41]$. Such findings suggest that the use of new, more accurate diagnostic approaches may complement clinical diagnostic procedures for select patients and help improve diagnostic accuracy in clinical practice, especially decreasing the rate of false positives. The goals of the current analysis were to analyze key variations between those with accurately diagnosed $\mathrm{AD}$ and false positives, but in order to test the full accuracy and value of such biomarkers samples must include not only "false positives" (e.g., the misdiagnosed group in this analysis) but also false negatives to establish both sensitivity and specificity. Moreover, it is likely there are important ramifications for those who are not diagnosed with probable or possible $\mathrm{AD}$ but are later found to have AD-related pathology (which is often mixed). This can serve as an important focus for future descriptive and clinical research on the health and cost outcomes of misdiagnosed AD.

The results need to be evaluated in light of several study limitations. First, it is unclear whether the findings can be generalized to patients evaluated in routine clinical practice as subjects in this study were assessed at National Institute on Aging Alzheimer's Disease Centers (ADCs) which are predominately urban, university medical centers that have recruited mostly (approximately 90\%) white participants [26]. Second, the current findings on potentially inappropriate medication use were based on a small sample size and will require replication. Data on the medical rationale for the choice of treatments were not available. Third, the high proportion of participants in the NACC-UDS with a likely AD diagnosis before enrollment in the NACC-UDS, the annual frequency of follow-up assessments in the NACC-UDS, the relatively small number of available assessments after the visit in which probable $\mathrm{AD}$ was first recorded, and the fact that medication use was recorded in the 2 weeks prior to a NACC-UDS assessment are among the other study limitations. Infrequent assessments indicate fewer opportunities to capture use of potentially unnecessary medications, suggesting that the current findings may 
have underestimated the true prevalence of such pharmacological therapies (as well as capturing use of appropriate medications). Last is the extensive missing data on the Geriatric Depression Scale which led to our use of less robust depression parameters (neither of which are empirically-validated measures of depression). The statistical differences we found on the single item measure of depression were reversed for those with available GDS data (due in part to those with severe dementia not completing the GDS in the NACC-UDS). Finally, the results should be considered in light of the fact that some of the data are based on self-report without the benefit of informant information to confirm diagnosis. As individuals who lived alone or were unmarried were more likely to be misdiagnosed, clinicians may not have had the same quality of data available for these participants.

\section{Conclusions}

This study highlights the importance of making an accurate diagnosis of $\mathrm{AD}$ in clinical practice (and especially ruling out $\mathrm{AD}$ ) in order to reduce potentially inappropriate treatment for patients' conditions. Additional research is required, however, to demonstrate the link between improved diagnostic accuracy and impaired patients' health outcomes. A greater understanding of the empirical associations between inappropriate treatment and adverse health outcomes in misdiagnosed AD patients would advance the current state-of-the-art of clinical AD research.

\section{Competing interests}

Joseph E. Gaugler, David L. Roth, and Tolulope Fafowora received funding from Eli Lilly and Company to complete this analysis. Haya Ascher-Svanum is an employee of Eli Lilly and Company. Andrew Siderowf is an employee of Avid Radiopharmaceuticals, Incorporated, which is a subsidiary of Eli Lilly and Company. Thomas G. Beach performs research services (payments to his research institute, not to him) as part of contractual agreements with Avid Radiopharmaceuticals/Eli Lilly Corporation, GE Healthcare, Piramal Healthcare and Navidea Biopharmaceuticals.

\section{Authors' contributions}

JEG had responsibility for writing, editing, and submitting the entire manuscript, oversight of data analysis, conceptualization of the study questions, and interpretation of results. HA-S had responsibility for writing and editing the manuscript, oversight of data analysis, conceptualization of the study questions and interpretation of results. DLR had primary responsibility for all data management and analysis. TF edited the manuscript and reviewed the clinical interpretation of the empirical results. AS edited the manuscript, provided input in analysis interpretation and presentation, and provided in-depth clinical expertise on inappropriate medication use in dementia. TB edited the manuscript and provided guidance on the use of his original data on misdiagnosis. All authors read and approved the final manuscript.

\section{Acknowledgement}

This study was supported by Eli Lilly and Company, Indianapolis, Indiana, USA. The NACC database is funded by National Institute on Aging Grant U01 AG016976.

\section{Sponsor's role}

The sponsor provided quality oversight of data analysis and interpretation prior to submission.

\section{Author details}

'Center on Aging, School of Nursing, University of Minnesota, Minneapolis, MN, USA. 'Lilly Research Laboratories, Eli Lilly and Company, Indianapolis, IN, USA. ${ }^{3}$ Center on Aging and Health, School of Medicine, Johns Hopkins University, Baltimore, MD, USA. ${ }^{4}$ Avid Radiopharmaceuticals Inc, Philadelphia, PA, USA. ${ }^{5}$ Banner Sun Health Research Institute, Sun City, AZ, USA.

Received: 15 July 2013 Accepted: 17 December 2013

Published: 19 December 2013

\section{References}

1. Grand JH, Caspar S, Macdonald SW: Clinical features and multidisciplinary approaches to dementia care. J Multidiscip Healthc 2011, 4:125-147.

2. Hyman BT, Phelps CH, Beach TG, Bigio EH, Cairns NJ, Carrillo MC, Dickson DW, Duyckaerts C, Frosch MP, Masliah E, Mirra SS, Nelson PT, Schneider JA, Thal DR, Thies B, Trojanowski JQ, Vinters HV, Montine TJ: National Institute on Aging-Alzheimer's Association guidelines for the neuropathologic assessment of Alzheimer's disease. Alzheimers Dement 2012, 8(1):1-13.

3. McKhann GM, Knopman DS, Chertkow H, Hyman BT, Jack CR Jr, Kawas CH, Klunk WE, Koroshetz WJ, Manly JJ, Mayeux R, Mohs RC, Morris JC, Rossor MN, Scheltens P, Carrillo MC, Thies B, Weintraub S, Phelps CH: The diagnosis of dementia due to Alzheimer's disease: recommendations from the National Institute on Aging-Alzheimer's Association workgroups on diagnostic guidelines for Alzheimer's disease. Alzheimers Dement 2011, 7(3):263-269.

4. Beach TG, Monsell SE, Phillips LE, Kukull W: Accuracy of the clinical diagnosis of Alzheimer disease at National Institute on Aging Alzheimer Disease Centers, 2005-2010. J Neuropathol Exp Neurol 2012, 71(4):266-273.

5. Lim A, Tsuang D, Kukull W, Nochlin D, Leverenz J, McCormick W, Bowen J, Teri L, Thompson J, Peskind ER, Raskind M, Larson EB: Cliniconeuropathological correlation of Alzheimer's disease in a communitybased case series. J Am Geriatr Soc 1999, 47(5):564-569.

6. Mayeux R, Saunders AM, Shea S, Mirra S, Evans D, Roses AD, Hyman BT, Crain B, Tang MX, Phelps CH: Utility of the apolipoprotein E genotype in the diagnosis of Alzheimer's disease. Alzheimer's disease centers consortium on apolipoprotein E and Alzheimer's disease. N Engl J Med 1998, 338(8):506-511.

7. Ranginwala NA, Hynan LS, Weiner MF, White CL III: Clinical criteria for the diagnosis of Alzheimer disease: still good after all these years. Am $J$ Geriatr Psychiatry 2008, 16((5):384-388.

8. Pearl GS: Diagnosis of Alzheimer's disease in a community hospital-based brain bank program. South Med J 1997, 90(7):720-722.

9. Klatka LA, Schiffer RB, Powers JM, Kazee AM: Incorrect diagnosis of Alzheimer's disease. A clinicopathologic study. Arch Neurol 1996, 53(1):35-42.

10. Piccini $C$, Bracco L, Amaducci L: Treatable and reversible dementias: an update. J Neurol Sci 1998, 153(2):172-181.

11. Hejl A, Hogh P, Waldemar G: Potentially reversible conditions in 1000 consecutive memory clinic patients. J Neurol Neurosurg Psychiatry 2002, 73(4):390-394.

12. Wang BW, Lu E, Mackenzie IR, Assaly M, Jacova C, Lee PE, Beattie BL, Hsiung GY: Multiple pathologies are common in Alzheimer patients in clinical trials. Can J Neurol Sci 2012, 39(5):592-599.

13. Gay BE, Taylor Kl, Hohl U, Tolnay M, Staehelin HB: The validity of clinical diagnoses of dementia in a group of consecutively autopsied memory clinic patients. J Nutr Health Aging 2008, 12(2):132-137.

14. Jellinger KA: Clinicopathological analysis of dementia disorders in the elderly-an update. J Alzheimers Dis 2006, 9(3 Suppl):61-70.

15. Brunnstrom H, Englund E: Clinicopathological concordance in dementia diagnostics. Am J Geriatr Psychiatry 2009, 17(8):664-670.

16. Boxer AL, Boeve BF: Frontotemporal dementia treatment: current symptomatic therapies and implications of recent genetic, biochemical, and neuroimaging studies. Alzheimer Dis Assoc Disord 2007, 21(4):S79-S87.

17. Irwin D, Lippa CF, Rosso A: Effects of prescribed medications on cognition and behavior in frontotemporal lobar degeneration. Am J Alzheimers Dis Other Demen 2010, 25(7):566-571.

18. Lomen-Hoerth C, Murphy J, Langmore S, Kramer JH, Olney RK, Miller B: Are amyotrophic lateral sclerosis patients cognitively normal? Neurology 2003, 60(7):1094-1097.

19. Lomen-Hoerth C, Anderson T, Miller B: The overlap of amyotrophic lateral sclerosis and frontotemporal dementia. Neurology 2002, 59(7):1077-1079. 
20. Kerchner GA, Tartaglia MC, Boxer A: Abhorring the vacuum: use of Alzheimer's disease medications in frontotemporal dementia. Expert Rev Neurother 2011, 11(5):709-717.

21. McKeith I, Mintzer J, Aarsland D, Burn D, Chiu H, Cohen-Mansfield J, Dickson D, Dubois B, Duda JE, Feldman H, Gauthier S, Halliday G, Lawlor B, Lippa C, Lopez OL, Machado JC, O'Brien J, Playfer J: Dementia with Lewy bodies. Lancet Neurol 2004, 3(1):19-28.

22. Baskys A: Lewy body dementia: the litmus test for neuroleptic sensitivity and extrapyramidal symptoms. J Clin Psychiatry 2004, 65(Suppl 11):16-22.

23. Shim YS, Roe CM, Buckles VD, Morris JC: Clinicopathologic study of Alzheimer's disease: Alzheimer mimics. J Alzheimers Dis 2013, 35(4):799-811.

24. Morris JC, Weintraub S, Chui HC, Cummings J, Decarli C, Ferris S, Foster NL, Galasko D, Graff-Radford N, Peskind ER, Beekly D, Ramos EM, Kukull WA: The Uniform Data Set (UDS): clinical and cognitive variables and descriptive data from Alzheimer Disease Centers. Alzheimer Dis Assoc Disord 2006, 20(4):210-216

25. Mirra SS, Heyman A, McKeel D, Sumi SM, Crain BJ, Brownlee LM, Vogel FS, Hughes JP, van Belle G, Berg L: The Consortium to Establish a Registry for Alzheimer's Disease (CERAD). Part II. Standardization of the neuropathologic assessment of Alzheimer's disease. Neurology 1991, 41(4):479-486.

26. McKhann G, Drachman D, Folstein M, Katzman R, Price D, Stadlan EM: Clinical diagnosis of Alzheimer's disease: report of the NINCDS-ADRDA work group under the auspices of Department of Health and Human Services Task Force on Alzheimer's disease. Neurology 1984, 34(7):939-944.

27. Braak H, Braak E: Neuropathological stageing of Alzheimer-related changes. Acta Neuropathol 1991, 82(4):239-259.

28. Folstein MF, Folstein SE, McHugh PR: Mini-Mental State: a practical method for grading the cognitive state of patients for the clinician. J Psychiatr Res 1975, 12:189-198.

29. Pfeffer RI, Kurosaki TT, Harrah CH Jr, Chance JM, Filos S: Measurement of functional activities in older adults in the community. J Gerontol 1982, 37(3):323-329.

30. Cummings JL, Mega M, Gray K, Rosenberg-Thompson S, Carusi DA, Gornbein J: The Neuropsychiatric Inventory: comprehensive assessment of psychopathology in dementia. Neurology 1994, 44(12):2308-2314.

31. Yesavage JA, Brink TL, Rose $T L$, Lum $O$, Huang $V$, Adey M, Leirer VO: Development and validation of a geriatric depression screening scale: a preliminary report. J Psychiatr Res 1982, 17(1):37-49.

32. Di Napoli M: Benefits of statins in cerebrovascular disease. Curr Opin Investig Drugs 2004, 5(3):295-305.

33. Mendez MF: Frontotemporal dementia: therapeutic interventions. Front Neurol Neurosci 2009, 24:168-178.

34. Hodges JR: Hope abandoned: memantine therapy in frontotemporal dementia. Lancet Neurol 2013, 12(2):121-123.

35. Lewy Body Dementia Association, Inc: Treatment options. http://www.lbda. org/content/treatment-options. Updated 2012. Accessed January 27th, 2013.

36. Rolinski M, Fox C, Maidment I, McShane R: Cholinesterase inhibitors for dementia with Lewy bodies, Parkinson's disease dementia and cognitive impairment in Parkinson's disease. Cochrane Database Syst Rev 2012, 3, CD006504.

37. SAS Institute Inc: Base SAS ${ }^{\circledR} 9.3$ Procedures Guide. Cary, NC: SAS Institute Inc 2011.

38. Bloudek LM, Spackman DE, Blankenburg M, Sullivan SD: Review and metaanalysis of biomarkers and diagnostic imaging in Alzheimer's disease. J Alzheimers Dis 2011, 26(4):627-645

39. Zhang S, Han D, Tan X, Feng J, Guo Y, Ding Y: Diagnostic accuracy of 18 FFDG and 11C-PIB-PET for prediction of short-term conversion to Alzheimer's disease in subjects with mild cognitive impairment. Int J Clin Pract 2012, 66(2):185-198
40. Grundman M, Pontecorvo MJ, Salloway SP, Doraiswamy PM, Fleisher AS, Sadowsky CH, Nair AK, Siderowf A, Lu M, Arora AK, Agbulos A, Flitter ML, Krautkramer MJ, Sarsour K, Skovronsky DM, Mintun MA, for the AV45-A17 Study Group: Potential impact of amyloid imaging on diagnosis and intended management in patients with progressive cognitive decline. Alzheimer Dis Assoc Disord 2013, 27(1):4-15.

41. Schipke CG, Peters O, Heuser I, Grimmer T, Sabbagh MN, Sabri O, Hock C Kunz M, Kuhlmann J, Reininger C, Blankenburg M: Impact of beta-amyloidspecific florbetaben PET imaging on confidence in early diagnosis of Alzheimer's disease. Dement Geriatr Cogn Disord 2012, 33(6):416-422.

doi:10.1186/1471-2318-13-137

Cite this article as: Gaugler et al.: Characteristics of patients misdiagnosed with Alzheimer's disease and their medication use: an analysis of the NACC-UDS database. BMC Geriatrics 2013 13:137.

\section{Submit your next manuscript to BioMed Central and take full advantage of:}

- Convenient online submission

- Thorough peer review

- No space constraints or color figure charges

- Immediate publication on acceptance

- Inclusion in PubMed, CAS, Scopus and Google Scholar

- Research which is freely available for redistribution
C Biomed Central 